Logic and Logical Philosophy

Volume 8 (2000), 47-63

Guido Vanackere

\title{
PREFERENCES AS INCONSISTENCY-RESOLVERS: The Inconsistency-adaptive Logic PRL
}

\begin{abstract}
In this paper I generalize the new approach to nonmonotonic reasoning that was presented in [6]. This generalization results in the inconsistency-adaptive logic PRL (PR stands for preference-based reliability strategy). I give proof theory, semantics, mention interesting properties, and comment on the reconstruction and amelioration of other nonmonotonic logics and mechanisms.
\end{abstract}

\section{Introduction}

Applying a paraconsistent logic to an inconsistent set of premises avoids triviality but results, in general, in a too poor consequence-set. An inconsistencyadaptive logic derives all 'rich' consequences where no inconsistency occurs and isolates inconsistencies. However, an inconsistency-adaptive logic does not resolve inconsistenties; the consequence-set will still contain inconsistencies. When we meet inconsistencies, in everyday life, we are interested in weeding out one half of each inconsistency. A straightforward way to do this, exists in tracking the sources where the inconsistent information derives from, and dropping the conclusion derived from the least reliable source.

The idea of annotating the premises according to their sources, is taken from [7], but the machinery of $\mathbf{P R L}$ is different. The annotated premise $A^{k}$ is a weakening of the 'normal' premise $A$ and is equated with $\sim^{k} \neg A$, in which, 
for $k=1,2, \ldots: \sim^{k}$ is a paraconsistent negation and $\neg$ is classical negation. I use letters as symbols for numbers, for the simple reason that this makes it easy to interpret the annotations as preferences. " $\sim^{k} \neg A$ " can be read as: "we give preference $k$ to the fact that we have no reason to reject $A$ ".

In this paper I do not question "Where do preferences on (the sources of) the premises come from?". In fact, PRL is an excellent logic even if the annotations are not interpreted as preferences. When preferences are known, the PRL-consequence-set is as strong and specific as we want it to be.

I give a quick overview of the results of the PRL-mechanism. Let $\Gamma^{A}$ be obtained from $\Gamma$ by annotating all members of $\Gamma$. Where $\mathbf{X L}$ is a logic, $C n_{\mathbf{X L}}(\Gamma)$ is the set of $\mathbf{X L}$-consequences of $\Gamma$, and $C n_{\mathbf{X L}}^{0}\left(\Gamma^{A}\right)$ the set of XL-consequences of $\Gamma^{A}$ in which no annotations occur. ${ }^{1}$ CL is Classical Logic. If $\Gamma$ is consistent, then $C n_{\mathbf{P R L}}^{0}\left(\Gamma^{A}\right)=C n_{\mathbf{C L}}(\Gamma)$. If $\Gamma$ is inconsistent, PRL reveals the conflicting premises. At this point, there are two possibilities: (1) It is not possible to interpret the annotations as preferences, i.e. it is not possible to introduce preferences on conflicting premises. In this case PRL 'deletes' both halves of each inconsistency. Let $\Gamma^{C} \subseteq \Gamma$ be the set of conflicting premises. Then $C n_{\mathbf{P R L}}^{0}\left(\Gamma^{A}\right)=C n_{\mathbf{C L}}\left(\Gamma-\Gamma^{C}\right)$. Obviously $C n_{\mathbf{C L}}(\Gamma)$ is trivial. (2) It is possible to introduce preferences on the conflicting premises. This is a non-logical step that can be made beforehand or at any stage of a proof. It is an advantage of PRL that we do not need to know the preferences of all premises. PRL reveals those premises on which the introduction of preferences will lead to the resolution of inconsistencies. If preferences of the conflicting premises are known, PRL deletes that half of each inconsistency that derives from the least preferred premises. Let $\Gamma^{P} \subset \Gamma^{C}$ be the set of conflicting premises that are not least preferred. In this case $C n_{\mathbf{P R L}}^{0}\left(\Gamma^{A}\right)=C n_{\mathbf{C L}}\left(\Gamma-\left(\Gamma^{C}-\Gamma^{P}\right)\right)$. Obviously $C n_{\mathbf{C L}}\left(\Gamma-\Gamma^{C}\right) \subseteq C n_{\mathbf{C L}}\left(\Gamma-\left(\Gamma^{C}-\Gamma^{P}\right)\right)$.

In section 2 , I give a brief characterisation of inconsistency-adaptives logics in general. In section 3, I give proof theory, semantics and metatheory of the lower limit logic pPRL. In section 4 , I do the same for PRL. In section 5, I comment on the influence of the formulation of the premises. In the 6th and last section, I try to show that PRL is a better nonmonotic tool than the (non-modal versions of) default logic. I also mention the possibility to reconstruct Rescher-mechanisms by means of PRL.

\footnotetext{
${ }^{1}$ Hence, $C n_{\mathbf{X L}}^{0}\left(\Gamma^{A}\right)$ does not contain formulas of the form $A^{k}, \sim^{k} \neg A$, or complex formulas containing such subformulas. All members of $C n_{\mathbf{X L}}^{0}\left(\Gamma^{A}\right)$ are 'normal' classically formalized formulas.
} 


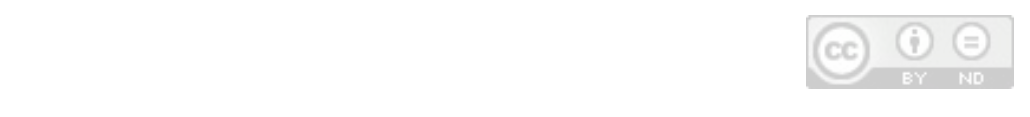

Preferences AS inCONSISTEnCY-RESOlVERS ...

\section{Inconsistency-adaptive logics in general}

Let IAL be any inconsistency-adaptive logic. IAL oscillates between a paraconsistent lower limit logic (PL) and a 'rich' upper limit logic (e.g. CL). Where no inconsistency occurs, IAL behaves like the upper limit logic; where an inconsistency occurs, IAL behaves like the lower limit logic. In [3] Diderik Batens argues that, in general, a weaker lower limit logic goes along with a richer IAL.

The question "how to get grip on "where an inconsistency occurs?" is formally answered by means of the minimal DEK-consequences (minimal disjunctions of (where necessary existentially quantified) contradictions that are $\mathbf{P L}$-derivable from the premises). In IAL some derivations are made on condition of the consistent behaviour of some involved (sub)formulas. The minimal DEK-consequences of the premises indicate which conditions are overruled. For instance, if $\Gamma \vdash_{\mathbf{P L}}(A \& \sim A) \vee(B \& \sim B) \vee(C \& \sim C)$, $\Gamma \nvdash_{\mathbf{P L}}(A \& \sim A) \vee(B \& \sim B), \Gamma \nvdash_{\mathbf{P L}}(A \& \sim A) \vee(C \& \sim C)$, and $\Gamma \nvdash_{\mathbf{P L}}$ $(B \& \sim B) \vee(C \& \sim C)$, then $(A \& \sim A) \vee(B \& \sim B) \vee(C \& \sim C)$ is a minimal DEK-consequence of $\Gamma$, and hence at least one of the formulas $A, B, C$ behaves inconsistently. Another example: suppose $q$ is derived from $\Gamma \cup\{\sim p, p \vee q\}$, on condition of the consistent behaviour of $p$. This condition is overruled if, e.g., $\Gamma \cup\{\sim p, p \vee q\} \vdash_{\mathbf{P L}} p \& \sim p$. In this case $p \& \sim p$ is a minimal DEK-consequence of $\Gamma \cup\{\sim p, p \vee q\}$ and thus $p$ behaves inconsistenly. Therefore $\Gamma \cup\{\sim p, p \vee q\} \nvdash_{\text {IAL }} q$.

There are two well-known strategies to select unreliable formulas among the factors of minimal DEK-consequences: ${ }^{2}$

1. Reliability. The consequence-set of $\Gamma$ is the set of formulas that are true in all models of $\Gamma$ in which the only true contradictions come from the factors of the minimal DEK-consequences of $\Gamma$. The factors of minimal DEK-consequences are the only unreliable formulas.

Example. If $p$ is derived from $\Gamma$ on condition of the consistent behaviour of $q$, and $(q \& \sim q) \vee(r \& \sim r)$ is a minimal DEK-consequence of $\Gamma$, then both $q$ and $r$ are unreliable and hence $p$ is not finally derivable from $\Gamma$.

2. Minimizing abnormality. The consequence-set of $\Gamma$ is the set of all formulas that are true in all minimally abnormal models of $\Gamma$, i.e. in all models of $\Gamma$ that verify a set of inconsistencies that is not a superset of a set of inconsistencies verified by another model of $\Gamma$.

${ }^{2}$ In [5], Kristof De Clercq develops two more strategies. 
Example. If $p$ is derived from $\Gamma$ on condition of the consistent behaviour of $q$, and $p$ is also derived from $\Gamma$ on condition of the consistent behaviour of $r$, and $(q \& \sim q) \vee(r \& \sim r)$ is a minimal DEK-consequence of $\Gamma$, then one type of minimally abnormal models verify $q \& \sim q$ and falsify $r \& \sim r$ whereas the other verify $r \& \sim r$ and falsify $q \& \sim q$. Hence all minimally abnormal models satisfy a condition on which $p$ is derivable. Therefore $p$ is finally derivable from $\Gamma$.

The reliability strategy has an easier proof-mechanism, but leads, in specific cases, to a poorer consequence-set than the minimal abnormality strategy. The latter difference however vanishes when preferences are taken into account. PRL is constructed by means of the reliability strategy.

\section{The underlying paraconsistent logic pPRL}

\subsection{Proof theory}

The language of $\mathbf{p P R L}$ is the language of classical predicate logic with identity, extended with an infinite set of paraconsistent negations $\sim^{1}, \sim^{2}$, .... The logic is obtained by adding to $\mathbf{C L}$ (with $\neg$ as the classical negation) the axiom schema $\left(\mathrm{A} \sim^{k}\right)$.

$$
\left(\mathrm{A} \sim^{k}\right) \quad A \vee \sim^{k} A \quad(\text { for } k=1,2, \ldots)
$$

Premises receive a preference $k \geq 1$ or an annotation that can be interpreted as a symbol for a preference (notation $A^{k}$ ). A higher number corresponds to a lower preference. Theorems receive preference 0 , the highest preference. Formally, the preferences are handled by equating $A^{0}$ with $A$, and $A^{k}$ with $\sim^{k} \neg A$. Substitution of identity is restricted as follows

$$
(\mathrm{A}=2) \quad \alpha=\beta \supset(A \supset B),
$$

where $B$ is obtained by replacing in $A$ an occurrence of $\alpha$ that occurs outside the scope of a paraconsistent negation by $\beta$.

In view of the weak interpretation of the premises, $\mathbf{p P R L}$ is an extremely poor logic. In a way of speaking it is not even possible to derive the premises. More exactly: $A^{k} \nvdash_{\text {pPRL }} A$. Moreover, $C n_{\mathbf{p P R L}}^{0}\left(\Gamma^{A}\right)=C n_{\mathbf{C L}} \emptyset$. If the statement, defended in [3] holds - a poorer lower limit logic goes along with a richer adaptive logic - pPRL must be an excellent lower limit logic.

In view of the axioms $\left(\mathrm{A} \sim^{k}\right)$ and $(\mathrm{A}=2)$, $\mathbf{p P R L}$ does not spread inconsistencies: no inconsistency entails another. PPRL shares this property with 
CLuN, the lower limit logic of the inconsistency-adaptive logics ACLuN1 and ACLuN2 (see [2]).

Let $!^{k} A$ stand for $A \& \sim^{k} A$ and let $\sharp^{k} A$ stand for $\neg A \& \sim^{k} \neg A$ (hence $!^{k} \neg A \equiv \sharp^{k} A$ ). The following theorem expresses a property of $\mathbf{p P R L}$ that is important in view of the construction of the adaptive logic PRL. (The easy proof is left to reader.)

Theorem 1 (Conditional Derivation CD).

$$
\Gamma^{A} \vdash_{\mathbf{p P R L}} \sim^{k} \neg A \quad \text { iff } \quad \Gamma^{A} \vdash_{\mathbf{p P R L}} \sharp^{k} A \vee A .
$$

$\mathrm{CD}$ can be used as a derivation rule in pPRL-proofs. This rule allows to derive the 'normal' interpretation $A$ of the premise $A^{k}$ in disjunction with $\sharp^{k} A$. In the adaptive logic PRL, $\sharp^{k} A$ is considered as false, unless and until proven otherwise. Therefore, it is right to talk about "conditional derivation": the normal interpretation of the premises is derivable on condition of the $\sim^{k}$-consistent behaviour of the classical negation of the premises.

\subsection{Semantics}

Let $\mathcal{S}$ be the set of sentential letters, $\mathcal{P}^{r}$ the set of predicative letters of rank $r, \mathcal{C}$ and $\mathcal{V}$ the set of letters for individual constants and variables respectively, $\mathcal{F}$ the set of all formulas (open and closed), and $\mathcal{N}=\left\{\sim^{k} A \mid\right.$ $A \in \mathcal{F}, k \geq 1\}$.

A pPRL-model is a couple $M=\langle D, v\rangle$ in which $D$ is a set and $v$ is an assignment-function defined by:

$\mathrm{S} 1.1 \quad v: \mathcal{S} \longrightarrow\{0,1\}$

$\mathrm{S} 1.2 \quad v: \mathcal{C} \cup \mathcal{V} \longrightarrow D$ is such that $D=\{v(\alpha) \mid \alpha \in \mathcal{C} \cup \mathcal{V}\}$

S1.3 $\quad v: \mathcal{P}^{r} \longrightarrow P\left(D^{r}\right)$ (the power set of the $r$-th Cartesian product of $D$ )

$\mathrm{S} 1.4 \quad v: \mathcal{N} \longrightarrow\{0,1\}$

The valuation-function $v_{M}$ determined by the model $M$ is defined as follows:

$\mathrm{S} 2.1 \quad v_{M}: \mathcal{F} \longrightarrow\{0,1\}$

S2.2 where $A \in \mathcal{S}, v_{M}(A)=v(A)$

$\mathrm{S} 2.3 \quad v_{M}\left(\pi^{r} \alpha_{1} \ldots \alpha_{r}\right)=1$ iff $\left\langle v\left(\alpha_{1}\right), \ldots, v\left(\alpha_{r}\right)\right\rangle \in v\left(\pi^{r}\right)$

S2.4 $v_{M}(\alpha=\beta)=1$ iff $v(\alpha)=v(\beta)$

S2.5 $v_{M}(\neg A)=1$ iff $v_{M}(A)=0$

S2.6 $\quad v_{M}\left(\sim^{k} A\right)=1$ iff $v_{M}(A)=0$ or $v\left(\sim^{k} A\right)=1$

S2.7 $v_{M}(A \supset B)=1$ iff $v_{M}(A)=0$ or $v_{M}(B)=1$

S2.8 $\quad v_{M}(A \& B)=1$ iff $v_{M}(A)=1$ and $v_{M}(B)=1$

S2.9 $v_{M}(A \vee B)=1$ iff $v_{M}(A)=1$ or $v_{M}(B)=1$ 
S2.10 $v_{M}(A \equiv B)=1$ iff $v_{M}(A)=v_{M}(B)$

S2.11 $v_{M}((\forall \alpha) A(\alpha))=1$ iff $v_{M}(A(\beta))=1$ for all $\beta \in \mathcal{C} \cup \mathcal{V}$

S2.12 $v_{M}((\exists \alpha) A(\alpha))=1$ iff $v_{M}(A(\beta))=1$ for at least one $\beta \in \mathcal{C} \cup \mathcal{V}$

Truth in a model, semantic consequence and validity are defined as usual.

\subsection{Metatheory}

The proofs of the soundness and completeness theorems are analogous to those for pHL2 (see [6]), and hence left to reader.

Theorem 2. $\Gamma^{A} \vdash_{\mathrm{pPRL}} A$ iff $\Gamma^{A} \vDash_{\mathrm{pPRL}} A$.

\section{PRL}

\subsection{Intuitive approach}

In order to get grip on the machinery of PRL, it is interesting to take a look at some pPRL-proofs.

\section{Proof 1.}

1. $(\forall x) P x^{k}$

$\begin{array}{ll}- & \text { PREM } \\ - & \text { PREM } \\ (1) & \text { DEF } \\ (2) & \text { DEF } \\ (3) & \text { CD } \\ (4) & \text { CD } \\ (5) & \text { UI } \\ (6,7) & \text { MP }^{3}\end{array}$

2. $(P a \supset p)^{m}$

3. $\quad \sim^{k} \neg(\forall x) P x$

PREM

4. $\sim^{m} \neg(P a \supset p)$

(1) DEF

5. $\sharp^{k}(\forall x) P x \vee(\forall x) P x$

(2) $\quad \mathrm{DE}$

6. $\sharp^{m}(P a \supset p) \vee(P a \supset p)$

(4) CD

7. $\sharp^{k}(\forall x) P x \vee P a$

$(6,7) \quad \mathrm{MP}^{3}$

\section{Proof 2.}

1. $(\forall x)(B x \supset F x)^{k}$

$\begin{array}{ll}- & \text { PREM } \\ - & \text { PREM } \\ - & \text { PREM }\end{array}$

2. $(\forall x)(P x \supset \neg F x)^{m}$

3. $\quad(\forall x)(P x \supset B x)^{n}$

PREM

${ }^{3}$ This application of Modus Ponens is derivable: From $A \vee(B \supset C)$ and $D \vee B$ we can derive by addition $A \vee D \vee(B \supset C)$ and $A \vee D \vee B$. From these formulas we can derive $A \vee D \vee((B \supset C) \& B)$ and from this follows $A \vee D \vee C$. This kind of sped up derivations is generally valid in $\mathbf{p P R L}$ :

$\left.\sharp C_{1} \vee \cdots \vee \sharp C_{m} \vee\left(\left(A_{1} \& . . \& A_{n}\right) \supset B\right), \sharp D_{1} \vee \cdots \vee \sharp D_{k} \vee\left(A_{1} \& . . \& A_{n}\right)\right) /$

$\sharp C_{1} \vee \cdots \vee \sharp C_{m} \vee \sharp D_{1} \vee \cdots \vee \sharp D_{k} \vee B$

The application of the instantiation rule UI in line (7) is an example of such a sped up derivation. 
4. $P a^{r}$

5. $\quad \sim^{k} \neg(\forall x)(B x \supset F x)$

6. $\quad \sim^{m} \neg(\forall x)(P x \supset \neg F x)$

7. $\quad \sim^{n} \neg(\forall x)(P x \supset B x)$

8. $\sim^{r} \neg P a$

9. $\sharp^{k}(\forall x)(B x \supset F x) \vee(\forall x)(B x \supset F x)$

10. $\sharp^{m}(\forall x)(P x \supset \neg F x) \vee(\forall x)(P x \supset \neg F x)$

11. $\sharp^{n}(\forall x)(P x \supset B x) \vee(\forall x)(P x \supset B x)$

12. $\sharp^{r} P a \vee P a$

$13 \sharp^{k}(\forall x)(B x \supset F x) \vee(B a \supset F a)$

$14 \sharp^{m}(\forall x)(P x \supset \neg F x) \vee(P a \supset \neg F a)$

$15 \sharp^{n}(\forall x)(P x \supset B x) \vee(P a \supset B a)$

16. $\sharp^{r} P a \vee \sharp^{m}(\forall x)(P x \supset \neg F x) \vee \neg F a$

17. $\sharp^{r} P a \vee \sharp^{n}(\forall x)(P x \supset B x) \vee B a$

18. $\sharp^{r} P a \vee \sharp^{n}(\forall x)(P x \supset B x) \vee \sharp^{k}(\forall x)(B x \supset F x) \vee F a$

$(13,17) \quad \mathrm{MP}$

(1) DEF

(2) DeF

(3) DEF

(4) DeF

(5) $\quad \mathrm{CD}$

(6) $\quad \mathrm{CD}$

(7) $\quad \mathrm{CD}$

(8) CD

(9) UI

(10) UI

(11) UI

$(12,14) \quad$ MP

$(11,12) \quad \mathrm{MP}$

19. $\sharp^{r} P a \vee \sharp^{m}(\forall x)(P x \supset \neg F x) \vee \sharp^{n}(\forall x)(P x \supset B x) \vee$

$$
\sharp^{k}(\forall x)(B x \supset F x) \vee(F a \& \neg F a)
$$

CONJ

20. $\neg(F a \& \neg F a)$

21. $\sharp^{r} P a \vee \sharp^{m}(\forall x)(P x \supset \neg F x) \vee \sharp^{n}(\forall x)(P x \supset B x) \vee \sharp^{k}(\forall x)(B x \supset F x)$

$(19,20) \quad$ DS

Whether the annotations are interpreted as preferences or not, has no influence on $\mathbf{p P R L}$-derivations. The difference shows up when we construct the adaptive logic PRL on pPRL. I illustrate the machinery of PRL, in comparison with $\mathbf{C L}$. Unless and until an inconsistency or a disjunction of inconsistencies is derived from the premises, PRL interprets a set of premises consistently. Hence, if no disjunction of inconsistencies is derived, all disjuncts of the form $\sharp^{k} C$ are false, and hence, for instance in proof 1 , $p$ is derivable from $\sharp^{k}(\forall x) P x \vee \sharp^{m}(P a \supset p) \vee p$.

If we apply $\mathbf{C L}$ or $\mathbf{P R L}$ - even without introducing preferences - to the first set of premises, we can derive $p$.

If we apply $\mathbf{C L}$ to the second set of premises, we derive triviality (both $F a$ and $\neg F a$ are derivable). If we apply PRL to the second set of premises, without interpreting the annotations as preferences, neither $F a$ nor $\neg F a$ are derivable (and hence we avoid triviality). If we apply PRL to the second set of premises, and we introduce preferences, then, (1) if $k>m, r$ or $n>$ $m, r$, then $\neg F a$ is derivable, but $F a$ not; (2) if $m>k, n, r$, then $F a$ is 
derivable, but $\neg F a$ not, and (3) if $r \geq k, m, n$, then neither $F a$ nor $\neg F a$ are derivable. $^{4}$

The formula in line (21) reveals the conflicting premises. If no premise is preferred, all premises mentioned in the formula in line (18) are considered as unreliable. If preferences are known, only the least preferred premise (it is, the premise annotated with the highest number) is unreliable. Indeed, PRL interprets a set of premises as consistent as possible. If a set of premises is inconsistent, a disjunction of inconsistencies is derivable. The minimal disjunctions of inconstencies (see exact definition below) indicate the unreliable premises. The introduction of preferences results in a smaller set of unreliable premises and hence in a richer consequence-set.

Where $\sharp^{k} A$ is a formula in which the variables $\alpha_{1}, \ldots, \alpha_{m}(m \geq 0)$ occur free, let $\exists \sharp^{k} A$ be $\left(\exists \alpha_{1}\right) \ldots\left(\exists \alpha_{m}\right)\left(\sharp^{k} A\right)$. Let $\operatorname{DENK}\left\{A_{1}^{k_{1}}, \ldots, A_{n}^{k_{n}}\right\}$ refer to $\exists \sharp^{k_{1}} A_{1} \vee \cdots \vee \exists \sharp^{k_{n}} A_{n}$, a disjunction of (where necessary) existentially quantified contradictions of classically negated premises. ${ }^{5} A_{1}^{k_{1}}, \ldots, A_{n}^{k_{n}}$ are the factors of the DENK-formula DENK $\left\{A_{1}^{k_{1}}, \ldots, A_{n}^{k_{n}}\right\}$.

Theorem 3. $\Gamma \vDash_{\mathbf{C L}} A$ iff there are $C_{1}^{k_{1}}, \ldots, C_{n}^{k_{n}} \in \mathcal{F}(0 \leq n)$ such that $\Gamma^{A} \vDash_{\text {pPRL }} \operatorname{DENK}\left\{C_{1}^{k_{1}}, \ldots, C_{n}^{k_{n}}\right\} \vee A$.

The proof is analogous to the proof of Theorems 6 and 7 in [6].

\subsection{Proof theory}

The idea of the proof theory of PRL is that we apply all rules derivable in pPRL unconditionally, whereas we equal every formula of the form $\sim^{k} \neg A$ with $A$ on condition that $\neg A$ behaves $\sim^{k}$-consistently.

Every line of a PRL-proof gets a fifth element in which we write the formulas on the consistent behaviour of which we rely to derive the formula in the second element by the rule mentioned in the fourth element from the formulas of the lines enumerated in the third element.

Definition. A occurs unconditionally at some line of a proof iff the fifth element of that line is empty.

\footnotetext{
${ }^{4}$ In contrast with PRL, HL2 (see [6]), assumes that 'descriptions of facts' do not come from unreliable sources. With PRL it is also possible to consider $P a$ as unreliable.

If $B$ stands for "is a bird", $F$ for "can fly", and $P$ for "is a penguin", it is natural to take $r, n<m<k$, and hence penguin $a$ cannot fly. Also: birds that are not penguins can fly.

${ }^{5}$ In view of the fact that the contradictions are contradictions of classically negated formulas, I speak of DENK instead of DEK (confer [2]). In section 5 it will become clear why it is necessary to assume that some disjunctions of contradictions have to be existentially quantified.
} 
Definition. A behaves $\sim^{k}$-consistently at a stage of a proof iff $\sharp^{k} A$ does not occur unconditionally in the proof at that stage.

Definition. The $\sim^{k_{1}}$-consistent behaviour of $A_{1}$ is connected to the $\sim^{k_{2}}$ consistent behaviour of $A_{2}, \ldots$, and the $\sim^{k_{n}}$-consistent behaviour of $A_{n}$ at a stage of a proof iff $\operatorname{DENK}\left\{A_{1}^{k_{1}}, \ldots, A_{n}^{k_{n}}\right\}$ occurs unconditionally in the proof at that stage whereas $\operatorname{DENK}\left\{A_{2}^{k_{2}}, \ldots, A_{n}^{k_{n}}\right\}$ does not occur unconditionally in it.

Definition. Where $A^{k}$ is a premise, the number $k$ is the preference of $A$.

Definition. If $A$ occurs as the second element of a line in a proof, the line preference of $A$ is the lowest preference (i.e. the highest number) of the formulas in the fifth element of that line. If the fifth element is empty, the line preference of $A$ is 0 .

Definition. The derivation preference of $A$ at a stage of a proof, is the highest line preference of $A$ at that stage of the proof. If $A$ does not occur as the second element of a line at that stage, the derivation preference is maximally low.

Definition. $A$ is reliable with respect to its preference at a stage of a proof, iff the preference of $A$ is higher than the derivation preference of $\sharp^{k} A$ at that stage of the proof. ${ }^{6}$

Definition. $A$ is reliable at a stage of a proof iff $A$ behaves $\sim^{k}$-consistently (for all $k$ ) at that stage and its consistent behaviour is not connected to the consistent behaviour of other formulas, or A is reliable with respect to its preference at that stage of the proof.

Given these definitions, proofs in PRL are governed by an unconditional rule, a conditional rule and a marking rule. The application of a rule produces the next stage.

RU All derivation rules of pPRL are unconditionally valid in any PRLproof. The fifth element of a new line is the union of the fifth elements of the lines mentioned in its third element.

RC From a line $(i)$ with $\operatorname{DENK}\left\{C_{1}^{k_{1}}, \ldots, C_{m}^{k_{m}}\right\} \vee A$ as second line, derive a new line with $A$ as second element, $(i)$ as third element, $\mathrm{RC}$ as fourth element and the union of $\left\{C_{1}^{k_{1}}, \ldots, C_{m}^{k_{m}}\right\}$ and of the fifth element of line $(i)$ as fifth element.

\footnotetext{
${ }^{6}$ If the derivation preference of $\sharp^{k} A$ is maximally low, $A$ is reliable with respect to its preference.
} 
RM If $C$ is not (anymore) reliable, then mark all lines the fifth element of which contains $C{ }^{7}$

At any stage of the proof, it is obligatory to apply RM and permitted to apply RU and RC. A marked line does not belong to the proof. If the fifth element of a line is empty, the formula in its second element is pPRLderivable from the premises and cannot be marked later. If the fifth element is not empty, its formula is provisionally derived. Unless it can also be derived at a line the fifth element of which is empty, it is not a pPRLconsequence. The unconditional occurrence of DENK-formulas in the proof determines which formulas are not anymore reliable.

As usual proofs may be sped up by derived rules. In general, I will use "conditional" rules as short cut rules for a combination of typical pPRLrules, $\mathrm{RC}$, and a classical derivation rule. "CUI", for instance, is the short cut rule for (1) $A^{k} / \sim^{k} \neg A$, (2) CD, (3) RC, and (4) UI.

A wff may be derived at some stage of a proof, while the line in which it occurs may be marked at a later stage of the proof. Therefore we need to distinguish between provisional and final consequences.

Definition. An extension of a PRL-proof is intelligent iff it has the following property: if both $\operatorname{DENK}(\Sigma)$ and $\operatorname{DENK}(\Sigma \cup \Pi)$ occur unconditionally in the extension, then the former precedes the latter.

Definition. $A$ is finally derived at some line in an PRL-proof iff it is the second element of that line and the line will not be marked in any intelligent extension of the proof.

Definition. $\Gamma^{A} \vdash_{\mathbf{P R L}} A, A$ is finally PRL-derivable from $\Gamma^{A}$, iff there is a PRL-proof from $\Gamma^{A}$ in which $A$ is finally derived.

It is possible to prove that $C n_{\mathbf{P R L}}\left(\Gamma^{A}\right)$ may be characterized without refering to the dynamics of the proofs. The characterization refers to $\mathbf{p P R L}$ only. The central point is that it depends only on pPRL-derivability (which is monotonic) whether a wff is reliable in an intelligent extension of the proof.

Lemma 1. If in an PRL-proof from $\Gamma^{A}, A$ occurs as the second element and $\left\{C_{1}^{k_{1}}, \ldots, C_{m}^{k_{m}}\right\}(0 \leq m)$ occurs as the fifth element of a line, then $\Gamma^{A} \vdash_{\text {pPRL }} A \vee \operatorname{DENK}\left\{C_{1}^{k_{1}}, \ldots, C_{m}^{k_{m}}\right\} .^{8}$

\footnotetext{
${ }^{7}$ As new lines in a proof take over the fifth elements of the lines they are derived from by means of $\mathrm{RC}$ or an application of $\mathrm{RU}$, it is obvious that lines derived from marked lines have to be marked too.

8 The proof of Lemma 1 is completely analogous to the proof of Lemma 2 in [2] and Lemma 2 in [6].
} 
This means that whenever $A$ is derived in a PRL-proof on condition of the consistent behaviour of $\left\{C_{1}^{k_{1}}, \ldots, C_{m}^{k_{m}}\right\}$, then $A \vee \operatorname{DENK}\left\{C_{1}^{k_{1}}, \ldots, C_{m}^{k_{m}}\right\}$ is PRL-derivable unconditionally.

Definition. A DENK-consequence of $\Gamma^{A}$ is a DENK-formula which is pPRL-derivable from $\Gamma^{A}$.

Definition. $\operatorname{DENK}(\Delta)$ is a minimal DEK-consequence of $\Gamma^{A}$ iff it is a DENK-consequence of $\Gamma^{A}$, and for no $\Phi \subset \Delta, \operatorname{DENK}(\Phi)$ is a DENK-consequence of $\Gamma^{A}$.

TheOREM 4. $\Gamma^{A} \vdash_{\text {PRL }} A$, iff there are $C_{1}^{k_{1}}, \ldots, C_{m}^{k_{m}} \in \mathcal{F}(0 \leq m)$ such that $\Gamma^{A} \vdash_{\mathrm{pPRL}} A \vee \mathrm{DENK}\left\{C_{1}^{k_{1}}, \ldots, C_{m}^{k_{m}}\right\}$, and none of $C_{1}^{k_{1}}, \ldots, C_{m}^{k_{m}}$ is a factor of a minimal DEK-consequence of $\Gamma$ unless it is reliable with respect to its preference. ${ }^{9}$

It follows from Theorem 4 that whenever $A$ occurs as the second element of a line of a PRL-proof with $\left\{C_{1}^{k_{1}}, \ldots, C_{m}^{k_{m}}\right\}$ as fifth element, a new line can be added with $A \vee \operatorname{DENK}\left\{C_{1}^{k_{1}}, \ldots, C_{m}^{k_{m}}\right\}$ as second element and an empty fifth element, and vice versa. If a classical contradiction is derived in a line with $\left\{C_{1}^{k_{1}}, \ldots, C_{m}^{k_{m}}\right\}$ as fifth element, a new line can be added with DENK $\left\{C_{1}^{k_{1}}, \ldots, C_{m}^{k_{m}}\right\}$ as second element and an empty fifth element. In PRL-proofs the latter derivation rule is called IDENK (introduction of a DENK-formula).

The following Theorem expresses an important feature of PRL:

Theorem 5. If $\Gamma^{A} \vdash_{\text {PRL }} A$, then it is possible to extend any proof from $\Gamma^{A}$ into a proof in which $A$ is finally derived from $\Gamma^{A} \cdot{ }^{10}$

I now list some derivable marking rules in PRL.

MR1. If DENK $\left\{C_{1}^{k_{1}}, \ldots, C_{m}^{k_{m}}\right\}$ occurs unconditionally as the second element of a line of a PRL-proof, then mark all lines the fifth element of which contains an unreliable factor of that DENK-formula.

MR2. If the derivation preference of $A$ (resp. $\neg A$ ) at line $(i)$ is lower than the derivation preference of $\neg A$ (resp. $A$ ) at any line of the proof, then mark line $(i)$.

Theorem 6. The marking rules MR1 and MR2 are derivable in PRL. ${ }^{11}$

\footnotetext{
9 The proof of Theorem 4 is completely analogous to the proof of Theorem 15 in [6].

10 The proof of Theorem 9 is completely analogous to the proof of Theorem 9 in [6].

11 The proofs for MR1 and MR2 are respectively analogous to the proofs of Theorems 10 and 15 in $[6]$
} 


\subsection{Semantics}

The PRL-semantics is obtained from the $\mathbf{p P R L}$-semantics by defining, for each $\Gamma^{A}$, a subset of the PRL-models of $\Gamma^{A}$. Any $\Gamma^{A}$ defines a set of semantically unreliable formulas. The PRL-models of $\Gamma^{A}$ are those pPRLmodels of $\Gamma^{A}$ in which only unreliable formulas behave inconsistently. The set of unreliable formulas with respect to $\Gamma^{A}$ is a subset of the factors of the minimal DENK-consequences of $\Gamma$.

Definition. If $\Gamma^{A} \vDash_{\mathbf{P R L}} \sim^{n} B$ (whereas $\Gamma^{A} \not \nvdash_{\mathbf{P R L}} \sim^{m} B(1 \leq m<n$ ), then $\mathrm{CP}_{\Gamma^{A}}(B)=n$. If $\Sigma=\left\{B_{1}^{k_{1}}, \ldots, B_{n}^{k_{n}}\right\}(n \geq 1)$, then $\mathrm{CP}_{\Gamma^{A}}(\operatorname{DENK}(\Sigma))$ is the maximum of $k_{1}, \ldots, k_{n}$.

Definition. If $B^{k}$ is a factor of the minimal DENK-consequence $\operatorname{DENK}(\Sigma)$ of $\Gamma^{A}$, and $\mathrm{CP}_{\Gamma^{A}}(B)=\mathrm{CP}_{\Gamma^{A}}(\Sigma)$, then $B^{k}$ is PRL-unreliable with respect to $\Gamma^{A} \cdot \mathcal{U}\left(\Gamma^{A}\right)$ is the set of all formulas that are $\mathbf{P R L}$-unreliable with respect to $\Gamma^{A}$.

Definition. Where $M$ is a pPRL-model, $\operatorname{ab}(\mathrm{M})=\left\{A \mid v_{M}\left(\exists \sharp^{k} A\right)=1\right\}$.

Definition. $M$ is a PRL-model of $\Gamma^{A}$ iff it is a pPRL-model of $\Gamma^{A}$ and $\mathrm{ab}(\mathrm{M}) \subseteq \mathcal{U}\left(\Gamma^{A}\right)$.

Definition. $\Gamma^{A} \vDash_{\mathbf{P R L}} A$ iff $A$ is true in all PRL-models of $\Gamma^{A}$.

\subsection{Metatheory}

The proofs of the soundness and completeness theorems for PRL are analogous to those for HL2 (see [6]).

Theorem 7. $\Gamma^{A} \vDash_{\text {PRL }} A$ iff $\Gamma^{A} \vdash_{\text {PRL }} A$

\subsection{Example}

\section{Proof 3. ${ }^{12}$}

\begin{tabular}{lllll} 
1. & $p^{j}$ & - & PREM & - \\
2. & $q^{k}$ & - & PREM & - \\
3. & $r^{l}$ & - & PREM & - \\
4. & $(p \supset \neg q)^{m}$ & - & PREM & - \\
\hline
\end{tabular}

\footnotetext{
${ }^{12}$ CSUB (conditional substitution) is the short cut rule for the combination of $A^{k} \vdash$ $\sim^{k} \neg A, \mathrm{CD}$ and $\mathrm{RC}$.
} 
Preferences as inCONSISTEnCy-Resolvers . . .

5. $(r \supset \neg q)^{n}$

6. $p$

7. $q$

8. $r$

9. $\quad p \supset \neg q$

10. $\quad r \supset \neg q$

11. $\neg q$

12. $\neg q$

13. $\neg p$

14. $\neg r$

15. $\sharp^{j} p \vee \sharp^{k} q \vee \sharp^{m}(p \supset \neg q)$

16. $\sharp^{k} q \vee \sharp^{l} r \vee \sharp^{n}(r \supset \neg q)$
(1)
PREM

CSub

CSub

CSub

CSub

CSUB $\quad(r \supset \neg q)^{n}$

MP $\quad p^{j},(p \supset \neg q)^{m}$

MP $\quad r^{l},(r \supset \neg q)^{n}$

MT $\quad q^{k},(p \supset \neg q)^{m}$

MT $\quad q^{k},(r \supset \neg q)^{n}$

IDENK

IDENK

If preferences are not known $p, q, r, p \supset \neg q$ and $r \supset \neg q$ are unreliable, and none of them is PRL-derivable. If, for instance, $k>j, l, m, n$, then $q$ is the only unreliable formula, and $p, r, p \supset \neg q, r \supset \neg q, \neg q \in C_{\mathbf{P R L}}^{0}\left\{p^{j}, q^{k}\right.$, $\left.r^{l},(p \supset \neg q)^{m},(r \supset \neg q)^{n}\right\}$. If $j>l>k, m, n$, then $p$ and $r$ are the only unreliable formulas, and $q, \neg p, \neg r, p \supset \neg q, r \supset \neg q \in C n_{\mathbf{P R L}}^{0}\left\{p^{j}, q^{k}, r^{l}\right.$, $\left.(p \supset \neg q)^{m},(r \supset \neg q)^{n}\right\}$.

\section{About the premises}

PRL-consequences are to a certain degree dependent of the formulation of the premises. However, if a premise is replaced by an equivalent premise, the consequence-set remains the same.

Example: $\Gamma^{A} \cup\left\{(p \supset q)^{k}\right\}$ and $\Gamma^{A} \cup\left\{(\neg p \vee q)^{k}\right\}$ have the same PRLconsequence-set.

When premises that have the same annotation (because they originate from the same source), are put together in one conjunction, the PRLconsequence-set changes drastically.

Example.

\begin{tabular}{|l||c|c|c|}
\hline \multicolumn{1}{|c||}{ Premises } & \multicolumn{3}{c|}{ Consequences } \\
\hline & $k<l$ & $k>l$ & $k=l$ \\
\hline \hline$A^{k}, B^{k}, \neg A^{l}$ & $A, B$ & $\neg A, B$ & $B$ \\
\hline$(A \& B)^{k}, \neg A^{l}$ & $A, B$ & $\neg A$ & - \\
\hline
\end{tabular}

Hence, if one is interested in tracking (and deleting) unreliable sources, one has to put all premises from one source in one conjunction. This results 
in a poorer consequence-set. If one is interested in tracking (and deleting) individual unreliable premises, one has to analyse the conjunctions.

If universally quantified formulas are premises, we can treat them in two ways. The first way is the way they are treated in the previous part of this paper. The second way is to consider them as (possibly infinite) lists of instances. Consider $(\forall x) A(x)^{k}$ as the short for $A(a)^{k}, A(b)^{k}, A(c)^{k}, \ldots$ This results in the following instantiation rule:

$$
(\forall x) A(x)^{k} \vdash_{(\mathbf{p}) \mathbf{P R L}} \sim^{k} \neg A(a) .
$$

In this case PRL includes HL2. Thus, it is not only possible to track unreliable universally quantified formulas, but also to track unreliable instances. This way, PRL avoids that a universally quantified formula becomes unreliable as soon as one exception occurs.

The following proof illustrates the richness of this version of PRL.

\section{Proof 4.}

$\begin{array}{lllll}\text { 1. } & (\forall x)(I x \supset W x)^{k} & - & \text { PREM } & - \\ \text { 2. } & (\forall x)(F x \supset W x)^{l} & - & \text { PREM } & - \\ \text { 3. } & (I a \vee F a)^{m} & - & \text { PREM } & - \\ \text { 4. } & (F b \& \neg W b)^{n} & - & \text { PREM } & - \\ \text { 5. } & I a \supset W a & (1) & \text { CUI } & (I a \supset W a)^{k} \\ \text { 6. } & F a \supset W a & (2) & \text { CUI } & (F a \supset W a)^{l} \\ \text { 7. } & F b \supset W b & (2) & \text { CUI } & (F b \supset W b)^{l} \\ \text { 8. } & I a \vee F a & (3) & \text { CSUB } & (I a \vee F a)^{m} \\ \text { 9. } & F b & (4) & \text { CSIM } & (F b \& \neg W b)^{n} \\ \text { 10. } & \neg W b & (4) & \text { CSIM } & (F b \& \neg W b)^{n} \\ \text { 11. } & W a & (5,6,8) & \text { DIL } & (I a \supset W a)^{k},(F a \supset W a)^{l}, \\ & & & & (I a \vee F a)^{m} \\ \text { 12. } & W b & (9,7) & \text { MP } & (F b \& \neg W b)^{n},(F b \supset W b)^{l}\end{array}$

If $I$ stands for "is Italian", $F$ stands for "is French", $W$ for "likes wine", and if premise (4) does not get a lower preference than premise (3), we might expect that $n \leq m<k=l$. Hence, in view of MR2, line (12) has to be marked, and hence, $\neg W b$ and $W a$ are finally derivable, whereas $W b$ is not.

With this interpretation of universally quantified formulas, some disjuncts of DENK-formulas can be existentially quantified. Whenever a formula $A(a)$ is derived from $(\forall x) A(x)^{k}$ by means of CUI, whereas a does not occur in the premises, the fifth element of the line in which $A(a)$ is 
derived will be the open formula $A(x)^{k}$. Hence it is possible that $A(x)^{k}$ is a factor of a DENK-formula $\operatorname{DENK}\left(\left\{A(x)^{k}\right\} \cup \Sigma\right)$, which is the short for $(\exists x) \sharp^{k} A(x) \vee \operatorname{DENK}(\Sigma)$.

\section{PRL and other nonmonotonic mechanisms}

\subsection{Default logic}

The definitions in this section, as well as the premises of Proofs 2 and 4 (above) are taken from [4].

A default theory is a pair $(D, W) . W$ is a set of first order formulas representing the facts which are known to be true with certainty. $D$ is a set of defaults of the form

$$
\frac{A: B_{1}, \ldots, B_{n}}{C}
$$

where $A, B_{i}$ and $C$ are classical formulas. The default has the intuitive reading: if $A$ is provable and for all $i(1 \leq i \leq n), \neg B_{i}$ is not provable, then derive $C$. Defaults with free variables are schemata representing all of their closed instances ([4] p. 41.).

In PRL 'defaults' can simply be formulated as formulas of the form $(A \supset C)^{k}$ and $(\forall x)(A(x) \supset C(x))^{k}$. Universally quantified formulas are considered as (possibly infinite) lists of instances. The (possibly infinite) list of formulas $B_{1}^{k_{1}}, B_{2}^{k_{2}}, \ldots$ does not need to be known in advance! Whenever $A$ (respectively $A(a)$ ) is derived with a derivation preference $l$, and hence $C$ (resp. $C(a)$ ) can be derived with a derivation preference $n$ which is the maximum of $k$ and $l$, and $\neg C$ (respectively $\neg C(a)$ ) is derived with a line preference $m$, the PRL-machinery guarantees that (1) $C$ (resp. $C(a))$ is derivable iff $n<m,(2) \neg C$ (resp. $\neg C(a))$ is derivable iff $m<n$, and (3) neither $C$ (resp. $C(a)$ ) nor $\neg C$ (resp. $\neg C(a)$ ) are derivable iff $n=m$. In any of these cases, PRL reveals the involved formulas $B_{i}$. If we are interested in reconstructing a default theory within $\mathbf{P R L}$, the preference of the formulas $B_{i}$ should not be higher than or equal to the preference of $A \supset C$. The members of $W$, the facts, can all get a preference 1 (this means that they can only be 'deleted' by theorems). The possibility to introduce an infinite number of preferences, allows to express a refined ordering on the PRL-equivalents of defaults.

Such a reconstruction is nothing but a special case of PRL. PRL allows even for the contradiction of facts. Actually, not all facts are true with certainty in all contexts. With the PRL-reinterpretation of the premises, it 
is not required to assume that $W$ is consistent. Generally, the machinery of PRL comes to the following 'default':

$C$ is derived with derivation preference $k: \neg C$ is not derived with line preference $l \leq k / C \in C n_{\mathbf{P R L}}^{0}\left(\Gamma^{A}\right)$.

The original version of default logic does not satisfy several "intended" properties. It is for instance not possible to derive Likes wine from (1) Italian: Likes Wine/Likes Wine, (2) French:Likes Wine/Likes Wine and (3) Italian $\vee$ French. As illustrated in Proof 4 (above) PRL derives this intended consequence.

At the other hand, default logic derives Usable(Left arm) \& Usable(Right arm) from (1) true: Usable $(x) \& \neg \operatorname{Broken}(x) / U s a b l e(x)$ and (2) Broken (Left arm) $\vee$ Broken(Right arm). In the following proof it is shown that PRL deals perfectly with these premises.

\section{Proof 4.}

1. $(\forall x)(U x)^{3}$

2. $(\forall x)(B x \supset \neg U x)^{2}$

3. $\quad(B l \vee B r)^{1}$

4. $U l$

5. $U r$

6. $\quad B l \supset \neg U l$

7. $B r \supset \neg U r$

8. $B l \vee B r$

9. $\neg U l \vee \neg U r$

10. $\neg U r$

11. $\neg U l$

PREM -

PREM -

PREM -

CUI $U l^{3}$

CUI $\quad U r^{3}$

CUI $\quad(B l \supset \neg U l)^{2}$

CUI $\quad(B r \supset \neg U r)^{2}$

CSub $\quad(B l \vee B r)^{1}$

DIL $\quad(B l \supset \neg U l)^{2},(B r \supset \neg U r)^{2}$, $(B l \vee B r)^{1}$

DS $\quad(B l \supset \neg U l)^{2},(B r \supset \neg U r)^{2}$, $(B l \vee B r)^{1}, U l^{3}$

DS $\quad(B l \supset \neg U l)^{2},(B r \supset \neg U r)^{2}$, $(B l \vee B r)^{1}, U r^{3}$

12. $\sharp^{2}(B l \supset \neg U l) \vee \sharp^{2}(B r \supset \neg U r)^{2} \vee \sharp^{1}(B l \vee B r) \vee \sharp^{3} U l$

$$
(5,10) \quad \text { IDENK - }
$$

13. $\sharp^{2}(B l \supset \neg U l) \vee \sharp^{2}(B r \supset \neg U r)^{2} \vee \sharp^{1}(B l \vee B r) \vee \sharp^{3} U r$

$(4,11) \quad$ IDENK -

In view of MR1 and the minimal DENK-consequences derived in lines (12) and (13) both $U l^{3}$ and $U r^{3}$ are unreliable, and hence lines (4), (5), (10) and (11) have to be marked. This means that none of $U l, U r, \neg U l, \neg U r$ are finally derivable. However $\neg U l \vee \neg U r$ is a PRL-consequence of the premises. 


\title{
6.2. Rescher's strong and weak consequences
}

Diderik Batens showed me, that if we apply the minimal abnormality strategy instead of the reliability strategy, (thus obtaining the logic PRL2), $C n_{\mathbf{P R L} 2}^{0}\left(\Gamma^{A}\right)$ is the set of Rescher's strong consequences of $\Gamma$. Indeed, $A$ is a PRL2-consequence of $\Gamma^{A}$ iff $A$ is true in all minimally abnormal models of $\Gamma^{A}$, whereas these minimally abnormal models verify the maximally consistent subsets of $\Gamma^{A}$. If $A$ is true in some minimally abnormal models of $\Gamma^{A}$, then $A$ is a weak consequence of $\Gamma^{A}$. I refer to Diderik Batens article in the Proceedings of the JS-Symposium (Torun, Poland, 1998) for the details.

\section{References}

[1] Batens, Diderik: "Inconsistency-adaptive logics and nonmonotonic logics", Logique et Analyse 145, March 1994, pp. 57-94.

[2] Batens, Diderik: "Inconsistency-adaptive logics", in Ewa Orłowska (ed.) Logic at Work. Essays Dedicated to the Memory of Helena Rasiowa. Heidelberg, NewYork, Physica Verlag, Springer, 1998, pp. 445-472.

[3] Batens, Diderik: "Rich inconsistency-adaptive logics. The clash between heuristic efficiency and realistic reconstruction", in François Beets \& Eric Gillet (eds.) Logique en perspective. Mélanges offert à Paul Gochet. Editions OUSIA 2000, pp. $513-543$.

[4] Brewka, G., J. Dix and K. Konolige, Nonmonotonic Reasoning. An Overview. CSLI Publications, Stanford California, 1997.

[5] De Clercq, Kristof: "Two new strategies for inconsistency-adaptive logics" (to appear).

[6] Vanackere, Guido, "HL2, an inconsistency-adaptive and inconsistency-resolving logic for general statements that might have exceptions", Journal of Applied Non-Classical Logics 10, 2000, pp. 317-338.

[7] Van Meirvenne, Joachim, "Indexed adaptive logics", Logique et Analyse 145, March 1994, pp. 41-55.

\author{
Guido Vanackere \\ Center for Logic and Philosophy of Science \\ University of Ghent, Belgium \\ Guido.Vanackere@rug.ac. be
}

